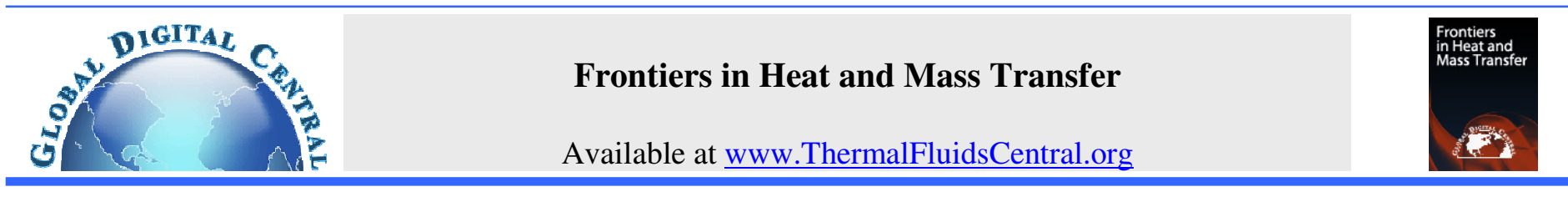

\title{
NATURAL CONVECTION ON A POROUS VERTICAL PLATE IN A DOUBLY STRATIFIED NON-DARCY POROUS MEDIUM
}

\author{
D. Srinivasacharya ${ }^{\mathrm{a},},{ }^{*}$ A.J. Chamkha ${ }^{\mathrm{b}}$, O. Surender ${ }^{\mathrm{a}}$, A.M. Rashad ${ }^{\mathrm{d}}$ \\ ${ }^{a}$ Department of Mathematics, National Institute of Technology, Warangal - 506004, India \\ ${ }^{b}$ Manufacturing Engineering Department, The Public Authority for Applied Education and Training, Shuweikh 70654, Kuwait \\ ${ }^{c}$ Department of Mathematics, Aswan University, Faculty of Science, 81528, Egypt \\ ${ }^{d}$ Department of Mathematics, Salman bin Abdul Aziz University, College of Science and Humanity Studies, AL-Kharj, Saudi Arabia
}

\begin{abstract}
The aim of the present article is to analyze the influence of thermal and mass stratification on natural convection heat and mass transfer over a porous vertical plate with uniform and constant wall temperature and concentration in porous medium. The Brinkman-Forchheimer based model is employed to describe the flow in the porous medium. The nonlinear governing equations and their associated boundary conditions are initially cast into dimensionless forms by pseudo-similarity variables. The resulting system of nonlinear, coupled partial differential equations is then solved numerically. The influence of pertinent parameters on the dimensionless velocity, temperature, concentration, heat and mass transfer coefficients are presented graphically.
\end{abstract}

Keywords: Thermal stratification, Solutal stratification, Suction/injection, Non-Darcy porous medium.

\section{INTRODUCTION}

The analysis of free convection heat and mass transfer in porous media has been an active field of research. It plays a crucial role in diverse applications, such as thermal insulation, distributions of temperature and moisture over agricultural fields and groves of fruit trees and damage of crops due to freezing and pollution of the environment, extraction of crude oil and chemical catalytic reactors, etc. The influence of suction and injection on convective heat and mass transfer over a vertical porous plate embedded in a porous medium has been the subject of many investigations owing to its relevance in many engineering and geophysical applications. Several researchers have studied natural convection heat and mass transfer over different geometries in porous medium. The influence of suction and injection on natural convective flow has been first studied by Eichhorn (1960). Soundalgekar et al. (1983) numerically investigated the problem of MHD free convection past a semi-infinite vertical plate with suction and injection. Pop and Watanabe (1992) studied the influence of suction or injection on steady free convection from a vertical cone. Makinde (2005) considered the combined free convection boundary layer flow past a permeable vertical plate with thermal radiation when the plate moves in its own plane. Alam et al. (2006) numerically studied the Dufour and Soret effects on unsteady MHD free convection and mass transfer flow past an infinite vertical porous plate embedded in a porous medium. Mbeledogu and Ogulu (2007) presented analytical closedform solution of the unsteady hydro-magnetic natural convection heat and mass transfer flow of a rotating, incompressible, viscous Boussinesq fluid in the presence of radiative heat transfer and a first order chemical reaction between the fluid and the diffusing species. Ibrahim et al. (2008) reported analytical solutions for heat and mass transfer by laminar flow of a Newtonian, viscous, electrically conducting and heat generation/absorbing fluid on a continuously vertical permeable surface in the presence of a radiation, a first-order homogeneous chemical reaction and the mass flux. Rajeshwari et al. (2009) considered the effect of chemical reaction on the forced and free convection boundary layer that flows in a semi-infinite expanse of an electrically conducting viscous incompressible fluid past vertical porous plate. Chamkha et al. (2010) investigated the effects of chemical reaction on unsteady free convective heat and mass transfer on a stretching surface in a porous medium. Singh et al. (2011) presented an analytical solution for three-dimensional free convection, laminar flow of an incompressible viscous fluid past an infinite, vertical, and porous plate embedded within a highly porous medium. Husnain et al. (2012) studied unsteady boundary layer flow and heat transfer characteristics of a viscous fluid through porous media in the case of variable viscosity and variable Prandtl number. Bhattacharyya et al. (2013) considered unsteady MHD boundary layer flow with diffusion of chemically reactive species undergoing first-order chemical reaction over a permeable stretching sheet with suction or blowing and also with power-law variation in wall concentration.

In all the above papers, the effect of stratification has been neglected. Stratification is a formation/ deposition of layers which occur due to temperature variations, concentration differences or the presence of different fluids. The analysis of natural convection in a doubly stratified medium is a fundamentally interesting and important problem because of its wide range of engineering applications. These applications include heat rejection into the environment such as lakes, rivers, and seas; thermal energy storage systems such as solar ponds; and heat transfer from thermal sources such as the condensers of power plants. It is important to examine the temperature stratification and concentration differences of hydrogen and oxygen in lakes and ponds as they may directly affect the growth rate of all cultured species. Also, the analysis of thermal stratification is important for solar engineering because higher energy efficiency can be achieved with better stratification. It has been shown by scientists that thermal stratification

*Corresponding author Email: dsc@ nitw.ac.in, dsrinivasacharya@yahoo.com 
in energy storage may considerably increase system performance. Although the effect of stratification of the medium on the heat removal process in a porous medium is important, very little work has been reported in literature.

Early studies on convection transport focused on seeking similarity solution because similar variables can give great physical insight with minimal efforts. However, the non-similarity boundary layer flows are more general in nature and in our everyday life, and thus are more important than the similarity ones. Hence, in the present study we have made an attempt to obtain non-similar solutions for the problem of natural convection on a vertical porous plate with constant suction/injection in a stable doubly stratified non-Darcian fluid saturated porous medium in which the ambient temperature and concentration varies linearly. The effects of pertinent parameters on dimensionless velocity, temperature, concentration, heat and mass transfer coefficients have been examined and presented graphically.

\section{MATHEMATICAL FORMULATION}

Consider the steady natural convection heat and mass transfer along a porous vertical plate embedded in a stable, doubly stratified viscous fluid saturated non-Darcy porous medium. Consider that the plate is subject to constant suction or injection $v_{0}$. The porous medium is considered to be homogeneous and isotropic (i.e., uniform with a constant porosity and permeability) and is saturated with a fluid which is in local thermodynamic equilibrium with the solid matrix. The fluid has constant properties except the density in the buoyancy term of the balance of momentum equation. The fluid flow is moderate, so the pressure drop is proportional to the linear combination of fluid velocity and the square of velocity (Forchheimer flow model is considered). Choose the coordinate system such that $\mathrm{x}$-axis is along the vertical plate and $y$-axis normal to the plate. The plate is maintained at uniform wall temperature $T_{w}$ and concentration $C_{w}$. The ambient medium is assumed to be vertically linearly stratified with respect to both temperature and concentration in the form $T_{\infty}(x)=T_{\infty, 0}+A_{1} x$ and $C_{\infty}(x)=C_{\infty, 0}+B_{1} x$ respectively, where $A_{1}$ and $B_{1}$ are constants varied to alter the intensity of stratification in the medium. The values of $T_{w}$ and $C_{w}$ are assumed to be greater than the ambient temperature $T_{\infty, 0}$ and concentration $C_{\infty, 0}$ at any arbitrary reference point in the medium (inside the boundary layer).

By employing laminar boundary layer flow assumptions, Boussinesq approximation, using the Brinkman-Forchheimer model and Dupuit Forchheimer relationship, the governing equations are given by.

$\frac{\partial u}{\partial x}+\frac{\partial v}{\partial y}=0$

$\frac{1}{\varepsilon^{2}}\left(u \frac{\partial u}{\partial x}+v \frac{\partial u}{\partial y}\right)=\frac{v}{\varepsilon} \frac{\partial^{2} u}{\partial y^{2}}+g \beta_{T}\left(T-T_{\infty}\right)+g \beta_{C}\left(C-C_{\infty}\right)-\frac{\mu}{\rho K_{p}} u-\frac{b}{K_{p}} u^{2}$

$u \frac{\partial T}{\partial x}+v \frac{\partial T}{\partial y}=\alpha \frac{\partial^{2} T}{\partial y^{2}}$

$u \frac{\partial C}{\partial x}+v \frac{\partial C}{\partial y}=D \frac{\partial^{2} C}{\partial y^{2}}$

where $u$ and $v$ are Darcy velocity components in $x$ and $y$ directions respectively, $T$ is the temperature, $C$ is the concentration, $g$ is the acceleration due to gravity, $\rho$ is the density, $b$ is the Forchheimer constant, $K_{p}$ is the permeability, $\varepsilon$ is the porosity, $\mu$ is the dynamic coefficient of viscosity, $\beta_{T}$ is the coefficient of thermal expansion, $\beta_{C}$ is the coefficient of solutal expansions, $\alpha$ is the effective thermal diffusivity and $D$ is the effective solutal diffusivity of the medium.

The boundary conditions are $u=0, \quad v=v_{0}, \quad T=T_{w}, \quad C=C_{w}, \quad$ at $\quad y=0$

$u=0, \quad T=T_{\infty}(x), \quad C=C_{\infty}(x) \quad$ as $\quad y \rightarrow \infty$

In view of the continuity Eq. (1), we introduce the stream function $\psi$ such that

$u=\frac{\partial \psi}{\partial y}, \quad v=-\frac{\partial \psi}{\partial x}$

Substituting Eqn. (6) in Eqs. (1)-(4) and then invoking the following non-similar transformations

$\xi=\frac{x}{L}, \eta=\frac{G r^{1 / 4}}{L \xi^{1 / 4}} y, \psi=\frac{\mu G r^{1 / 4} \xi^{3 / 4}}{\rho} f(\xi, \eta)$

$T-T_{\infty}(x)=\left(T_{w}-T_{\infty, 0}\right) \theta(\xi, \eta), C-C_{\infty}(x)=\left(C_{w}-C_{\infty, 0}\right) \phi(\xi, \eta)$

where $T_{\infty}$ and $C_{\infty}$ are ambient temperature and concentration resp. and $\alpha, D$ are thermal and solutal diffusivity resp.

Substituting the above transformations in Eqs. (1) - (4), we obtain the following nonlinear system of partial differential equations

$$
\begin{aligned}
& \frac{1}{\varepsilon} f^{\prime \prime \prime}+\frac{3}{4 \varepsilon^{2}} f f^{\prime \prime}-\frac{1}{2} f^{\prime 2}+\theta+B \phi-\frac{\xi^{1 / 2}}{D a G r^{1 / 2}} f^{\prime}-\frac{F_{c}}{D a} \xi f^{\prime 2} \\
& =\frac{\xi}{\varepsilon^{2}}\left[f^{\prime} \frac{\partial f^{\prime}}{\partial \xi}-f^{\prime \prime} \frac{\partial f}{\partial \xi}\right] \\
& \frac{1}{P r} \theta^{\prime \prime}-\varepsilon_{1} \xi f^{\prime}+\frac{3}{4} f \theta^{\prime}=\xi\left[f^{\prime} \frac{\partial \theta}{\partial \xi}-\theta^{\prime} \frac{\partial f}{\partial \xi}\right] \\
& \frac{1}{S c} \phi^{\prime \prime}-\varepsilon_{2} \xi f^{\prime}+\frac{3}{4} f \phi^{\prime}=\xi\left[f^{\prime} \frac{\partial \phi}{\partial \xi}-\phi^{\prime} \frac{\partial f}{\partial \xi}\right]
\end{aligned}
$$

The boundary conditions are transformed as follows

$$
\begin{aligned}
& f^{\prime}(\xi, 0)=0,3 f(\xi, 0)+4 \xi\left(\frac{\partial f}{\partial \xi}\right)_{\eta=0}=\gamma \xi^{1 / 4} \\
& \theta(\xi, 0)=1-\varepsilon_{1} \xi, \phi(\xi, 0)=1-\varepsilon_{2} \xi \text { at } \eta=0 \\
& f^{\prime}(\xi, \infty)=0, \theta(\xi, \infty)=0, \phi(\xi, \infty)=0 \text { as } \eta \rightarrow \infty
\end{aligned}
$$

where the prime denotes differentiation with respect to $\eta, \gamma$ is suction/ injection parameter,

$G r=\frac{g \beta_{T}\left(T_{w}-T_{\infty, 0}\right) L^{3}}{v^{2}}$ is the Grashof number, $\operatorname{Pr}=\frac{v}{\alpha}$ is the Prandtl number, $S c=\frac{v}{D}$ is the Schmidt number, $F_{c}=\frac{b}{L}$ is the Forchheimer number, $D a=\frac{K_{p}}{L^{2}}$ is the Darcy number, $B=\frac{\beta_{C}\left(C_{w}-C_{\infty, 0}\right)}{\beta_{T}\left(T_{w}-T_{\infty, 0}\right)}$ is the buoyancy ratio, $\quad \varepsilon_{1}=\frac{A_{1} L}{\left(T_{w}-T_{\infty, 0}\right)} \quad$ and $\quad \varepsilon_{2}=\frac{B_{1} L}{\left(C_{w}-C_{\infty, 0}\right)} \quad$ are the thermal and solutal stratification parameters.

Results of practical interest are skin friction coefficient, heat and mass transfer rates. The local skin friction coefficient $C_{f}$, local Nusselt number $N u_{\xi}$ and the local Sherwood number $S h_{\xi}$, are, respectively given by

$$
G r^{1 / 4} C_{f}=2 \xi^{-3 / 4} f^{\prime \prime}(\xi, 0), \frac{N u_{\xi}}{G r^{1 / 4}}=-\xi^{3 / 4} \theta^{\prime}(\xi, 0), \frac{S h_{\xi}}{G r^{1 / 4}}=-\xi^{3 / 4} \phi^{\prime}(\xi, 0)
$$

\section{NUMERICAL METHOD}

Equations (8) to (10) represent an initial-value problem with $\xi$ playing the role of time. This general non-linear problem cannot be solved in closed form and, therefore, a numerical solution is necessary to describe 
the physics of the problem. The implicit, tri-diagonal finite-difference method similar to that discussed by Blottner (1970) has proven to be adequate and sufficiently accurate for the solution of this kind of problems. Therefore, it is adopted in the present work. All first-order derivatives with respect to $\xi$ are replaced by two-point backwarddifference formulae when marching in the positive $\xi$ direction. Then, all second-order differential equations in $\eta$ are discretized using threepoint central difference quotients. This discretization process produces a tri-diagonal set of algebraic equations at each line of constant $\xi$ which is readily solved by the well known Thomas algorithm (see Blottner (1970)). During the solution, iteration is employed to deal with the nonlinearity aspect of the governing differential equations. The problem is solved line by line starting with line $\xi=0$ where similarity equations are solved to obtain the initial profiles of velocity, temperature and concentration and marching forward in $\xi$ until the desired line of constant $\xi$ is reached. Variable step sizes in the $\eta$ direction with $\Delta \eta_{1}=0.001$ and a growth factor $G=1.035$ such that $\Delta \eta=G \Delta \eta_{1}$ and constant step sizes in the $\xi$ direction $\Delta \xi=0.01$ are employed. These step sizes are arrived at after many numerical experimentations performed to assess grid independence. The convergence criterion employed in the present work is based on the difference between the current and the previous iterations. When this difference reached $10^{-5}$ for all points in the $\xi$ directions, the solution was assumed converged and the iteration process was terminated. All the computations were carried out for fixed values of $\varepsilon=0.8, B=1.0, P r=0.71, G r=5.0, S c=0.22, F_{c}=1.0 \quad$ and $D a=1.0$, $\varepsilon_{1}=0.1, \varepsilon_{2}=0.3, \gamma=0.1$. Some of the effects of the parameters on the physical quantities are very low (almost negligible) and hence they are not presented graphically.

\section{RESULTS AND DISCUSSION}

The non-dimensional velocity of the fluid falls near the plate and increases away from the plate with the rise of Forchheimer number as shown in Fig. 1. Since $F_{c}$ represents the inertial drag, an increase in the Forchheimer number enhances the resistance to the flow and so a decrease in the fluid velocity near the plate ensues. Here $F_{c}=0$ represents the case where the flow is Darcian. The velocity is maximum in this case due to the total absence of inertial drag. We notice from Fig. 2 that the temperature of the fluid diminishes with the rise of thermal stratification parameter. When thermal stratification is taken into consideration, the effective temperature difference between the plate and the ambient fluid will decrease; therefore, the thermal boundary layer is thickened and the temperature is reduced. Fig. 3 reveals that the concentration of the fluid decreases with the increase of

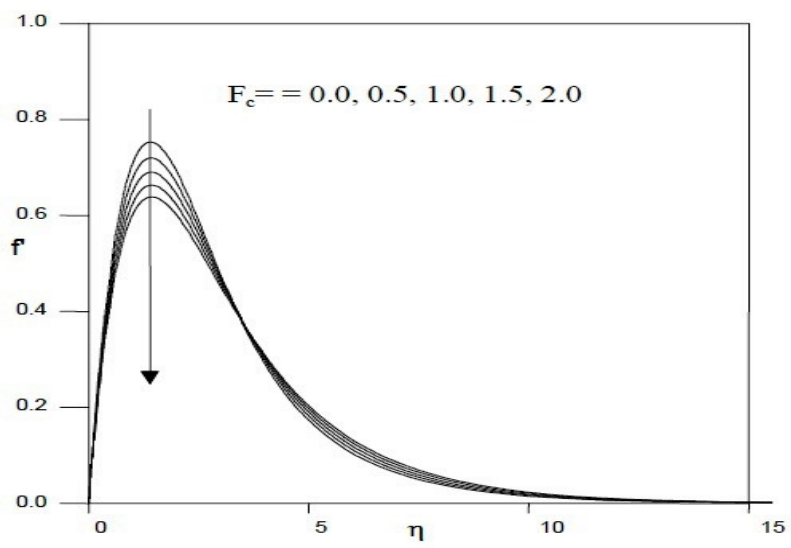

Fig. 1 Non-dimensional velocity profiles for different values of Forchheimer number the mass stratification parameter. It is observed that the nondimensional temperature and concentration values would become negative inside the boundary layer for different values of the stratification parameters depending on the values of other parameters.

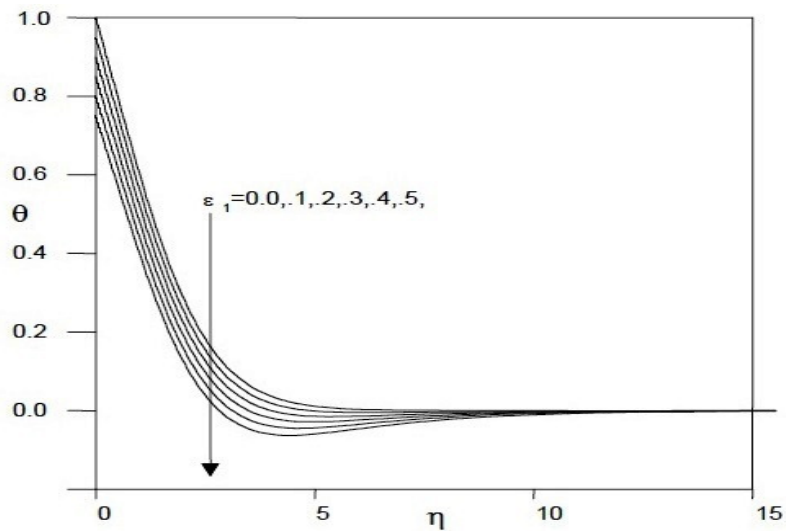

Fig. 2 Non-dimensional temperature profiles for different values of thermal stratification parameter

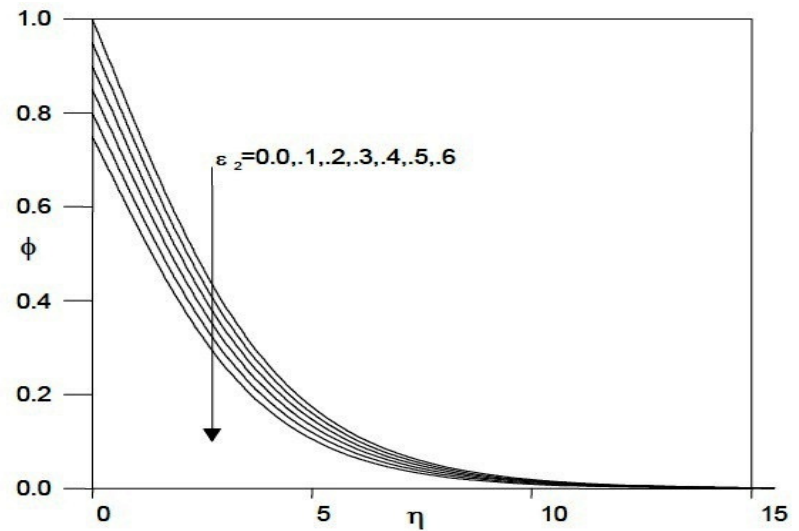

Fig. 3 Non-dimensional concentration profiles for different values of solutal stratification parameter

The influence of Darcy number $D a$ on non-dimensional velocity, temperature and concentration profiles with $\eta$ is presented in Figs. 4-6. Figure 4 shows that the velocity of the fluid is enhanced with the hike of Darcy number. The dimensionless temperature and concentration of the fluid decrease with the rise of Darcy number as shown in Figs. 5 and 6 respectively.

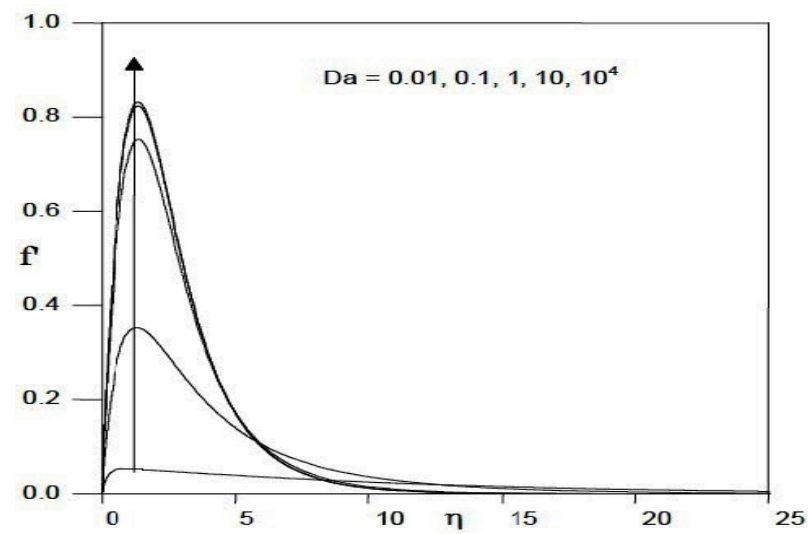

Fig. 4 Non-dimensional velocity profiles for different values of Darcy number 


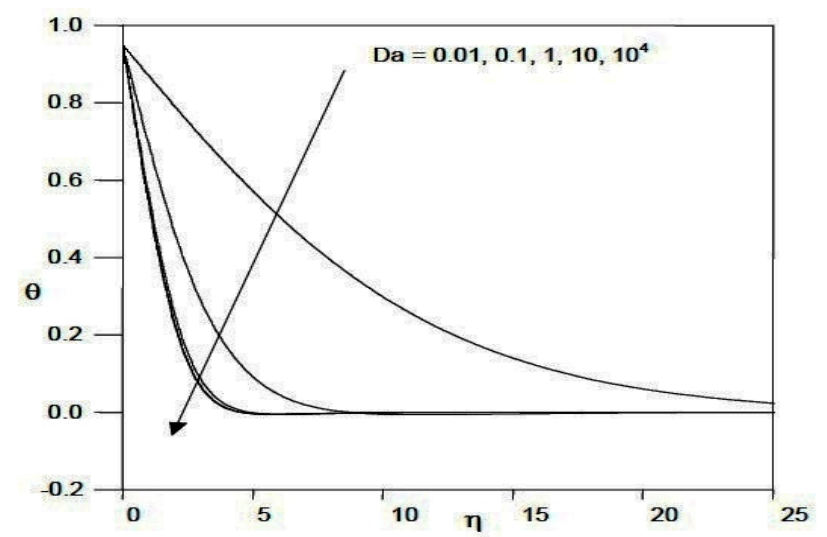

Fig. 5 Non-dimensional temperature profiles for different values of Darcy number

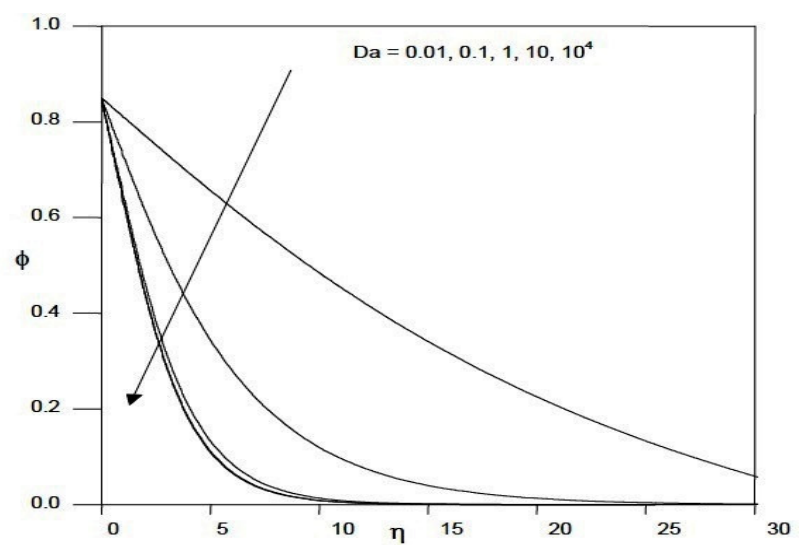

Fig. 6 Non-dimensional concentration profiles for different values of Darcy number

An increase in the intensity of suction/injection parameter causes a significant enhancement in the non-dimensional velocity, temperature and concentration in the boundary layer as shown in Figs. 7-9.

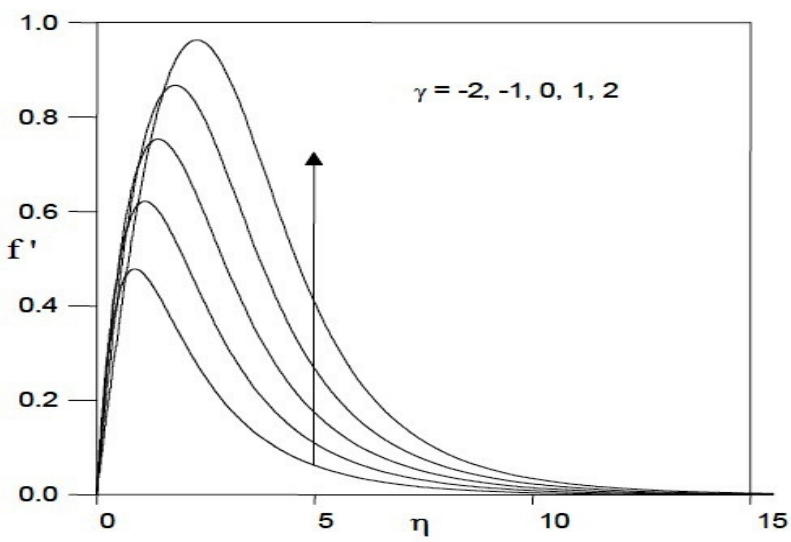

Fig. 7 Non-dimensional velocity profiles for different values of suction/injection parameter

The local heat and mass transfer coefficients (Nusselt number $\mathrm{Nu}$ and Sherwood number $S h_{\xi}$ ) for different values of thermal and solutal stratification parameters are presented in Figs. 10-11. Figure 10 shows that the non-dimensional local heat transfer rate decreases with the increase in the value of thermal stratification parameter $\varepsilon_{1}$. Figure 11 illustrates that the local mass transfer coefficient decrease with the increase in the thermal stratification parameter. This is due to the effective mass transfer between the plate and the ambient medium decreases as the thermally stratified effect increases. The dimensionless local heat and mass transfer coefficients are reduced with the rise of solutal stratification parameter $\varepsilon_{2}$ as shown in Figs. 12-13.

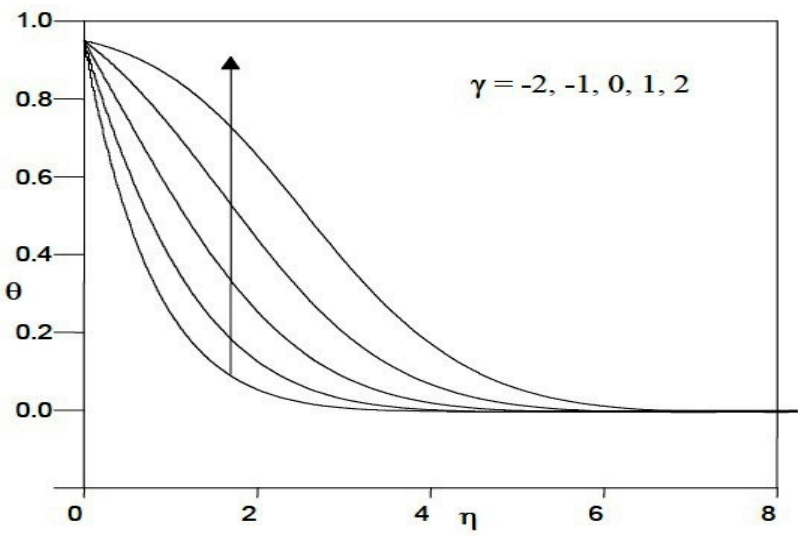

Fig. 8 Non-dimensional temperature profiles for different values of suction/injection parameter

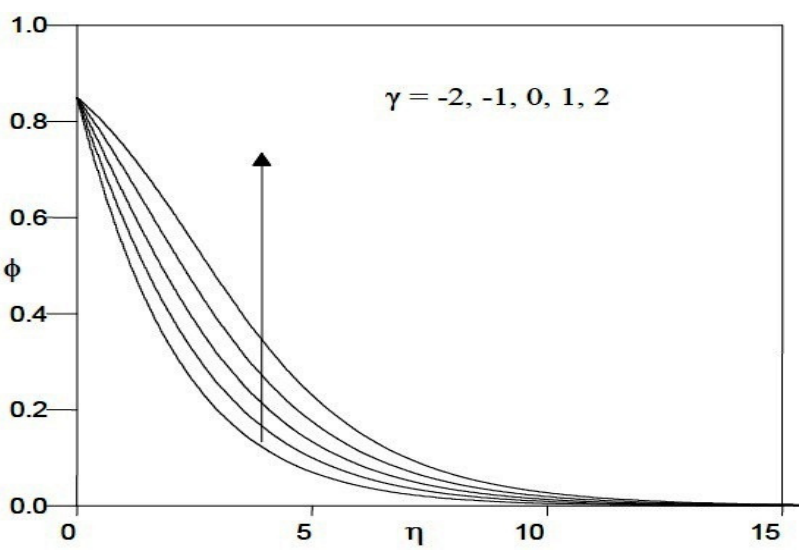

Fig. 9 Non-dimensional concentration profiles for different values of suction/injection parameter

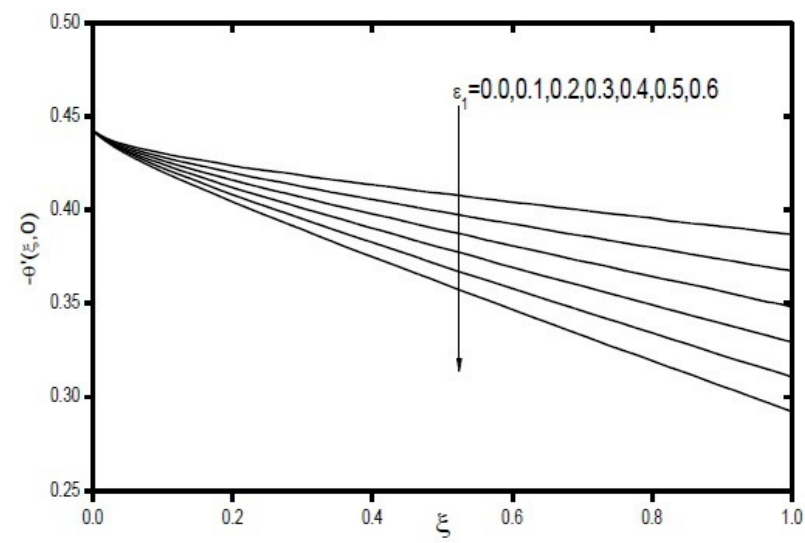

Fig. 10 Non-dimensional heat transfer rate for different values of thermal stratification parameter

Both local heat and mass transfer rates decreases with the increase in Forchheimer number $F_{c}$ as shown in Figs. 14 and 15. Since $F_{c}$ represent the inertial drag, thus an increase in the Forchheimer number retards the flow and hence diminishes the dimensionless heat and mass transfer rates in the boundary layer. 


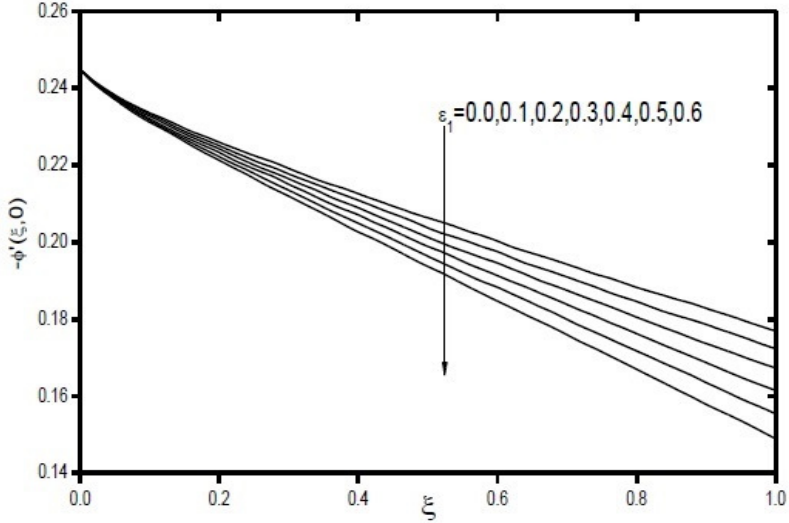

Fig. 11 Non-dimensional mass transfer rate for different values of thermal stratification parameter

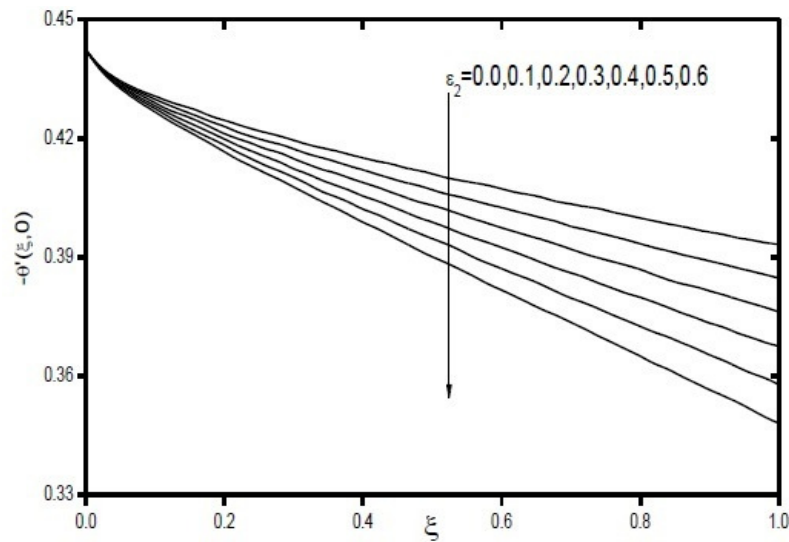

Fig. 12 Non-dimensional heat transfer rate for different values of solutal stratification parameter

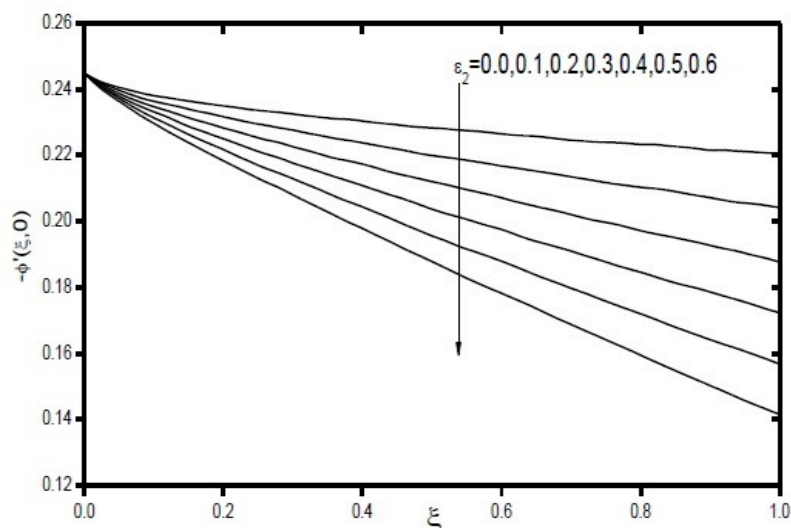

Fig. 13 Non-dimensional mass transfer rate for different values of solutal stratification parameter

The influence of suction/injection parameter $\gamma$ on local heat and mass transfer coefficients is shown in Figs. 16-17. An increase in the value of suction/injection parameter causes a significant decrease in the local heat and mass transfer coefficients as depicted in Figs. 16 and 17.

The dimensionless skin friction coefficient is appreciably decreased with the increase of thermal and solutal stratification parameters, Forchheimer number and suction/injection parameter as shown in Figs. 18-21

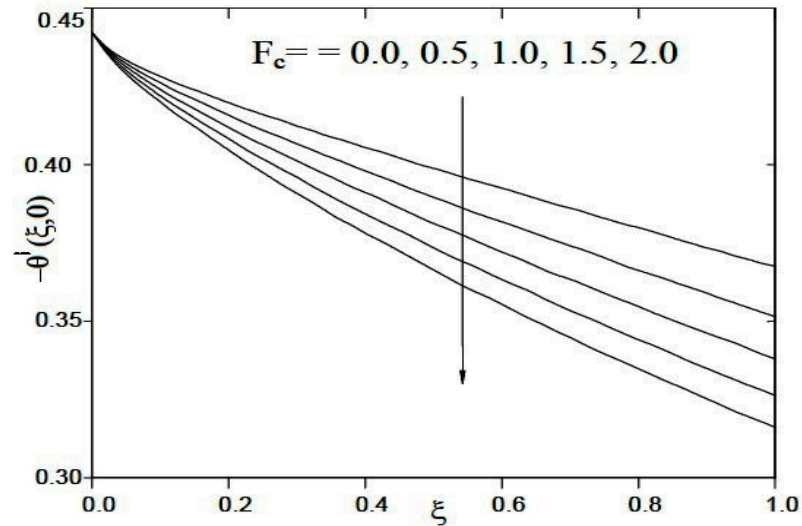

Fig. 14 Non-dimensional heat transfer rate for different values of Forchheimer number.

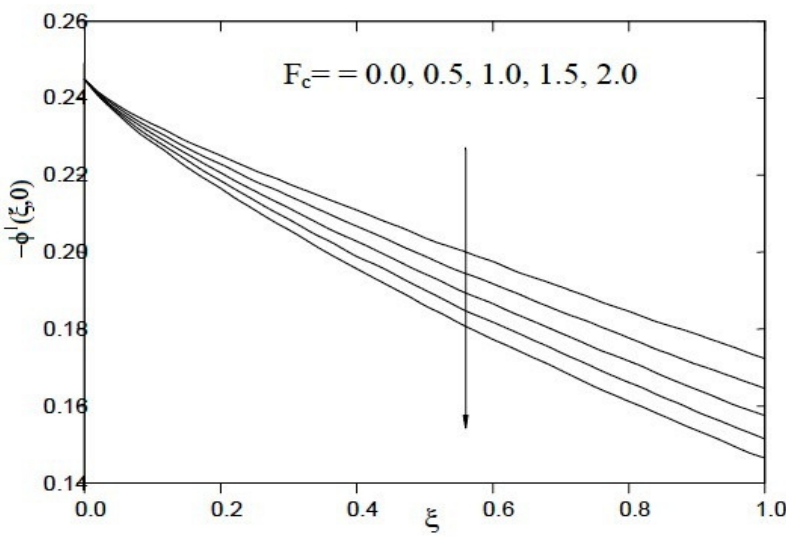

Fig. 15 Non-dimensional mass transfer rate for different values of Forchheimer number

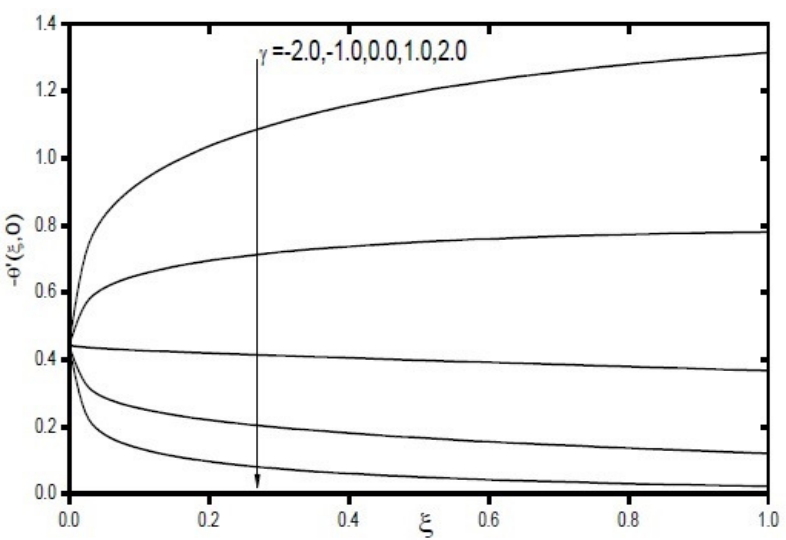

Fig. 16 Non-dimensional heat transfer rate for different values of suction/injection parameter.

\section{CONCLUSIONS}

In this article, the effect of double stratification on natural convection heat and mass transfer over a vertical porous plate in a fluid saturated non-Darcy porous medium with constant suction/injection has been analyzed using boundary layer approximation. The plate is maintained at uniform and constant wall temperature and concentration. The governing non-dimensional partial differential equations are solved numerically using Blottner method. 


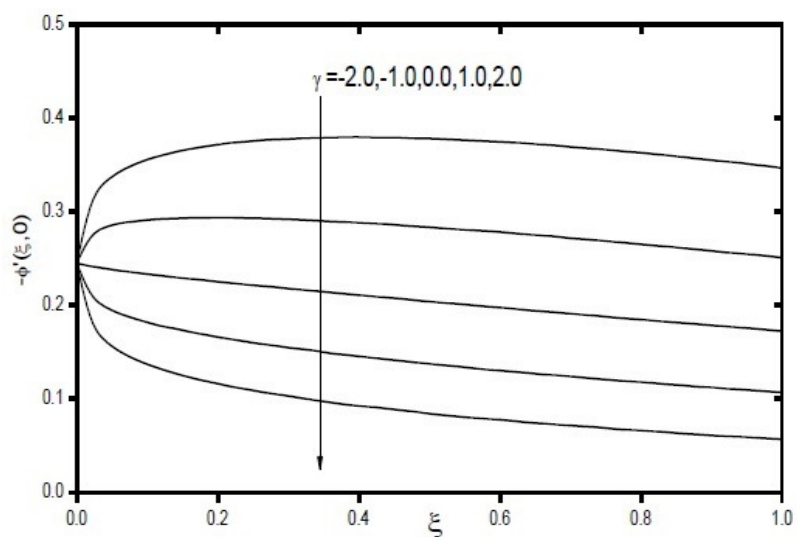

Fig. 17 Non-dimensional mass transfer rate for different values of suction/injection parameter.

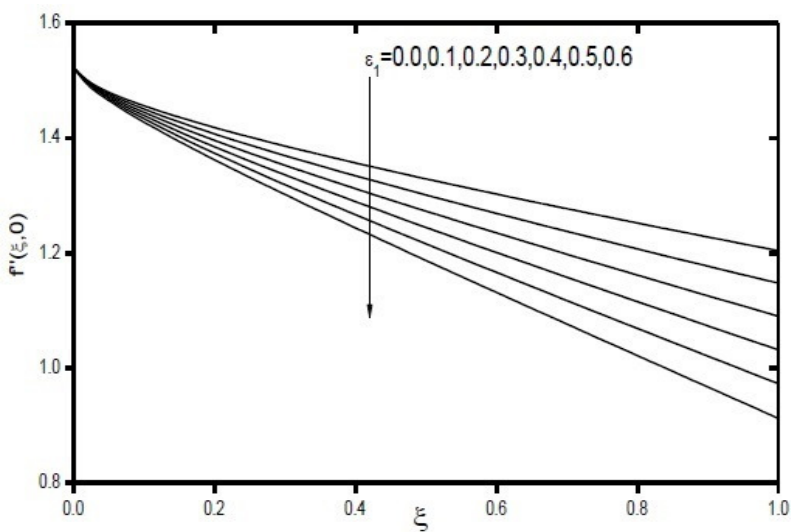

Fig. 18 Non-dimensional skin friction coefficient for different values of thermal stratification parameter

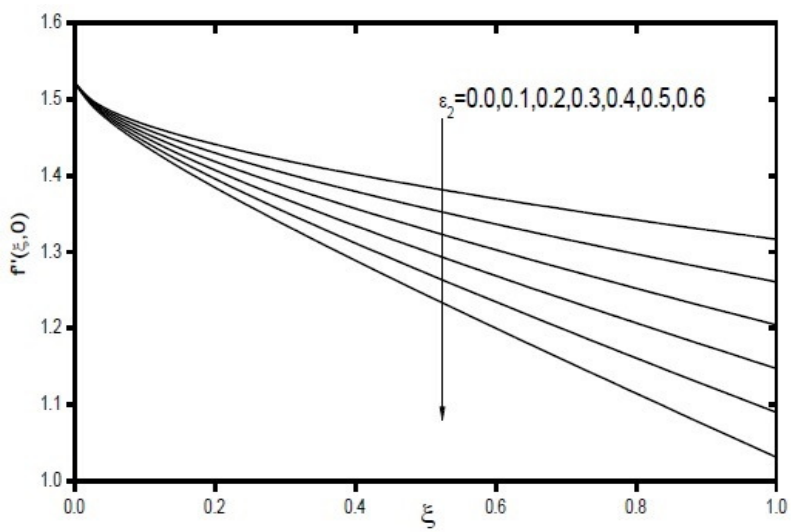

Fig. 19 Non-dimensional skin friction coefficient for different values of solutal stratification parameter

From the above study, we conclude that

1. An increase in thermal stratification parameter, decreases temperature distributions, non-dimensional heat and mass transfer coefficients, and skin friction coefficient.

2. An increase in mass stratification parameter reduces concentration distributions, non-dimensional heat and mass transfer coefficients in the boundary layer.

3. The higher value of Darcy number results in lower velocity but the higher temperature, concentration in the boundary layer.

4. The non-dimensional velocity of the fluid decreases near the plate and increases away from the plate with the increase of Forchheimer number. The higher value of Forchheimer number results in lower local heat and mass transfer rates.
5. An increase in the intensity of suction/injection parameter significantly enhanced the non-dimensional velocity, temperature and concentrations in the boundary layer but reduced both local heat and mass transfer rates.

6. The dimensionless skin friction coefficient is notably decreased with the increase of stratification parameters, Forchheimer number and suction/injection parameter.

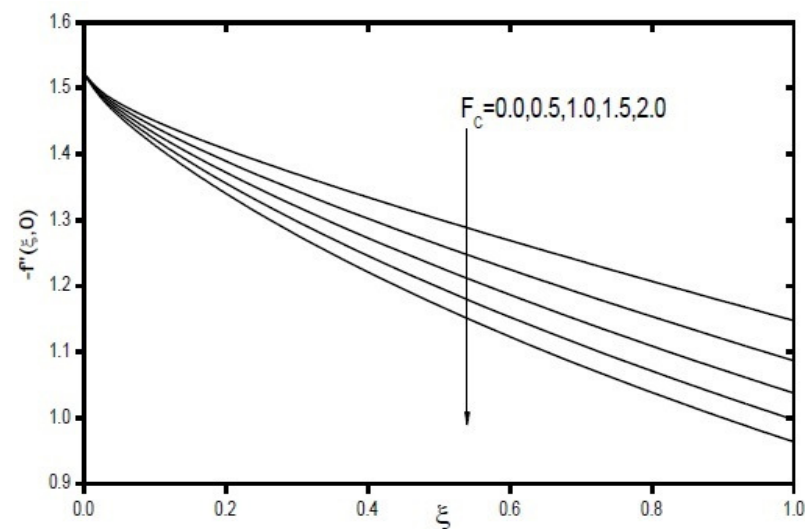

Fig. 20 Non-dimensional skin friction coefficient for different values of Forchheimer number

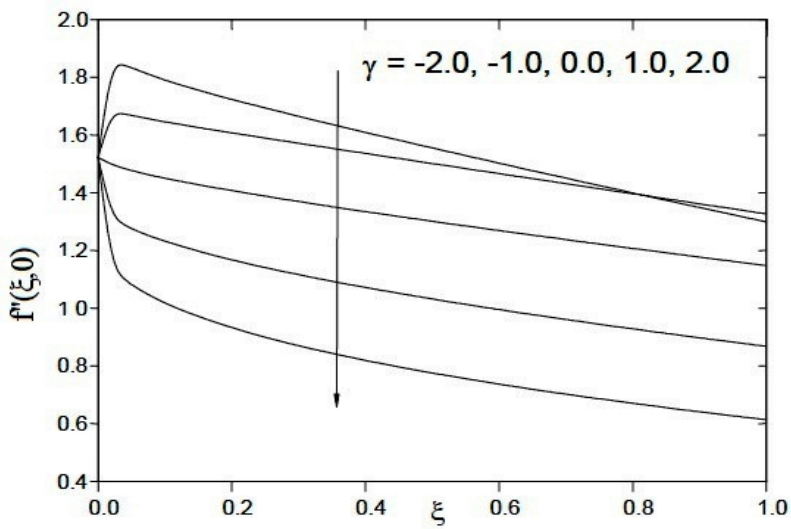

Fig. 21 Non-dimensional skin friction coefficient for different values of suction/injection parameter

\section{REFERENCES}

Alam, M., Rahman, M., and Samad, M., 2006, "Dufour and Soret Effects on Unsteady MHD Free Convection and Mass Transfer Flow Past a Vertical Porous Plate in a Porous Medium," Nonlinear Analysis: Modelling and Control, 11(3), 217-226.

Bhattacharyya, K., Mukhopadhyay, S., and Layek, G., 2013, "Unsteady MHD Boundary Layer Flow with Diffusion and First-Order Chemical Reaction Over a Permeable Stretching Sheet With Suction or Blowing," Chemical Engineering Communications, 200, 379-397. http://dx.doi.org/10.1080/00986445.2012.712577

Blottner, F., 1970, "Finite-difference Methods of Solution of the Boundary Layer Equations," AIAA Journal, 8, 193-205. http://dx.doi.org/10.2514/3.5642

Chamkha, A., Aly, A., and Mansour, M., 2010, "Similarity Solution for Unsteady Heat and Mass Transfer From a Stretching Surface Embedded in a Porous Medium with Suction/ Injection and Chemical Reaction Effects," Chemical Engineering Communications, 197, 846-859. http://dx.doi.org/10.1080/00986440903359087 
Husnain, S., Mehmood, A., Beg, O., and Ali, A., 2012, "Suction and Blowing Effects on Unsteady Flow and Heat Transfer Through Porous Media with Variable Viscosity," Journal of Porous Media, 15(3), 293202. http://dx.doi.org/10.1615/JPorMedia.v15.i3.70

Ibrahim, F., Elaiw, A., and Bakr, A., 2008, "Effect of the Chemical Reaction and Radiation Absorption on the Unsteady MHD Free Convection Flow Past a Semi Infinite Vertical Permeable Moving Plate with Heat Source and Suction," Communications in Nonlinear Science and Numerical Simulation, 13, 1056-1066. http://dx.doi.org/10.1016/j.cnsns.2006.09.007

Makinde, O., 2005, "Free Convection Flow with Thermal Radiation and Mass Transfer Past a Moving Vertical Porous Plate," International communications in Heat and Mass Transfer, 32, 1411-1419. http://dx.doi.org/10.1016/j.icheatmasstransfer.2005.07.005

Mbeledogu, I., and Ogulu, A., 2007, "Heat and Mass Transfer of an Unsteady MHD Natural Convection Flow of a Rotating Fluid Past a Vertical Porous Flat Plate in the Presence of Radiative Heat Transfer," International Journal of Heat and Mass Transfer, 50, 1902-1908. http://dx.doi.org/10.1016/j.ijheatmasstransfer.2006.10.016
Pop, I., and Watanabe, T., 1992, "Free Convection with Uniform Suction or Injection From a Vertical Cone for Constant Wall Heat Flux, "International Communications in Heat and Mass Transfer, 19, 275283. http://dx.doi.org/10.1016/0735-1933(92)90038-J

Rajeshwari, R., Jothiram, B., and Nelson, V., 2009, "Chemical Reaction, Heat and Mass Transfer on Nonlinear MHD Boundary Layer Flow Through a Vertical Porous Surface in The Presence of Suction," Applied Mathematical Sciences, 3, 2469-2480.

Singh, A., Singh, P., and Singh, N., 2011, "Effects of Periodic Permeability and Suction Velocity on Three-Dimensional Free Convection Flow Past a Vertical Porous Plate Embedded in Highly Porous Medium," Journal of Porous Media, 14(5), 451-460. http://dx.doi.org/10.1615/JPorMedia.v14.i5.70

Soundalgekar, V., Singh, M., and Takhar, H., 1983, "MHD Free Convection Past a Semi-Infinite Vertical Plate with Suction and Injection," Nonlinear Analysis, Theory, Methods \& Applications, 7(9), 941-944. 